\title{
Failed thoracic endografting of a mycotic aneurysm of the descending thoracic aorta
}

\author{
Nadia Hakkenbrak ${ }^{1}$, Maarten Truijers $^{2}$, and Irene Thomassen ${ }^{2}$ \\ ${ }^{1}$ VU Medisch Centrum \\ ${ }^{2}$ Spaarne Gasthuis
}

October 29, 2020

\begin{abstract}
An 82-year old male was referred with dyspnea and fever after a Staphylococcus aureus bacteraemia. The CTA scan showed a mycotic aneurysm of the descending thoracic aorta with impending rupture. Antibiotic treatment was started and endovascular aorta repair performed. Postoperatively, patient deteriorated as result of severe tracheal compression due to progressive dilatation of the aneurysm caused by a suspected type I endoleak. A tracheal stent was placed and the endovascular stent graft extended. Despite decreased ventilation pressure patient died of respiratory insufficiency. Even though, antibiotic treatment and endovascular stent grafting have improved outcome of mycotic aneurysms, severe complications following unsuccessful endovascular stent grafting do occur and can be lethal.
\end{abstract}

Failed thoracic endografting of a mycotic aneurysm of the descending thoracic aorta

Nadia A.G. Hakkenbrak ${ }^{1}$, Maarten Truijers ${ }^{1}$, Irene Thomassen ${ }^{1}$

Department of vascular surgery, Amsterdam UMC, location VU medical centre, the Netherlands

\section{Corresponding author:}

N.A.G. Hakkenbrak

Amsterdam UMC, location VUMC

Department of Surgery

De Boelelaan 1117

1081 HV, Amsterdam, The Netherlands

Email: n.hakkenbrak@amsterdamumc.nl

Tel: $+31-20-4444400$

Fax: $+31-20-4444512$

\section{Compliance with Ethical standards}

Funding: This study was not supported by any funding.

Conflict of Interest: The authors declare that they have no conflict of interest.

Ethical approval: This article does not contain any studies with human participants performed by any of the authors.

Informed consent: Informed consent was obtained from the patients representative. 
Consent to publication/Data availability statement: family/representative agreed to publishing of the case details and images.

Consent for publication: Consent for publication was obtained for every individual person's data included in the study.

\section{Abstract}

An 82-year old male was referred with dyspnea and fever after a Staphylococcus aureus bacteraemia. The CTA scan showed a mycotic aneurysm of the descending thoracic aorta with impending rupture. Antibiotic treatment was started and endovascular aorta repair performed. Postoperatively, patient deteriorated as result of severe tracheal compression due to progressive dilatation of the aneurysm caused by a suspected type I endoleak. A tracheal stent was placed and the endovascular stent graft extended. Despite decreased ventilation pressure patient died of respiratory insufficiency.

Even though, antibiotic treatment and endovascular stent grafting have improved outcome of mycotic aneurysms, severe complications following unsuccessful endovascular stent grafting do occur and can be lethal.

Key words: mycotic aneurysm, endovascular stent grafting, respiratory insufficiency, mortality.

\section{Introduction}

Mycotic aortic aneurysms are a rare presentation of aortic disease, taking account of less than $1 \%$ of all aneurysms in the Western World ${ }^{1,2}$. Conventional treatment consists of open aneurysm repair, however, this has become less popular due to favourable outcomes of endovascular stent grafting ${ }^{4,5}$. In this case report we describe a severe and lethal course following endovascular endografting of a mycotic thoracic aneurysm.

\section{Case description}

An 82- year old male was admitted to the hospital with fever and joint pain of the right knee, thumb and left shoulder. Blood, urine and bursa cultures of the thumb and shoulder showed a Staphylococcus aureus bacteremia. Based on these cultures intravenous flucloxacillin was prescribed, six gram/day. His medical history revealed an ischemic cerebral vascular accident, psoriasis vulgaris and corneal transplant for which he used acetylsalicylic acid, dipyridamole and valacyclovir.

After two weeks of intravenous antibiotic treatment a chest X-ray was performed on suspicion of a pneumonia (CRP $180 \mathrm{mg} / \mathrm{l}$, leukocytes $13 \times 10^{9} / \mathrm{l}$ ). The X-ray showed no signs of a pneumonia but, however, widening of the mediastinum. An additional computed tomography angiography scan (CTA scan) showed impending rupture of a mycotic saccular aneurysm of the descending thoracic aorta of $55 \mathrm{~mm}$, four centimeters below the left subclavian artery, with compression of the trachea and esophagus (Figure 1).

Therefore, patient was referred to our tertiary referral center. At arrival patient was hemodynamically stable but suffered from dyspnea and a subtle expiratory stridor. There were no signs of dysphagia, hypoxemia or fever. An emergency thoracic endovascular aortic repair (TEVAR) procedure was performed; a Gore ${ }^{\odot} \mathrm{CTAG}^{\circledR} 37 \mathrm{~mm} \times 10 \mathrm{~cm}$ was expanded just distal of the left subclavian artery. Completion angiography showed exclusion of the aneurysm without signs of endoleak.

Several hours following the procedure the patient deteriorated, suffering from tachycardia, tachypnea and respiratory failure. A CTA scan was performed, showing progressive compression of the trachea and esophagus. The diameter of the saccular aneurysm was stable $(56 \mathrm{~mm})$ and a type $1 \mathrm{~b}$ endoleak, inadequate distal sealing, was suspected (Figure 2). Emergency bronchoscopy showed severe obstruction of the distal tracheal lumen, carina, proximal left and right bronchus. A silicone Dumon ${ }^{\circledR}$ stent $(18 \times 14 \times 14 \mathrm{~mm})$ was deployed in the trachea. Because of persistent compression an additional fully covered expandable Microtech ${ }^{\circledR}$ nitinol Y stent $(16 \times 13 \times 13 \mathrm{~mm})$ was placed to allow mechanical ventilation. The aortic stent graft was extended to both ends with a Gore ${ }^{\complement}$ CTAG ${ }^{\circledR} 37 \mathrm{~mm} \times 20 \mathrm{~cm}$ just below the left common carotid artery, overstenting the subclavian artery. Completion angiography showed exclusion of the aneurysm (Figure 3). Although the 
operation did result in decreased ventilation pressure and even detubation, the patient died four days after surgery because of excess pulmonary secretion and subsequent respiratory insufficiency.

\section{Discussion}

The majority of the mycotic aortic aneurysms (MAA) develop as result of a bacteraemia in a patient with a pre-existing atherosclerotic aortic aneurysm ${ }^{1}$. This may be due to seeding of disrupted aortic intima or mural thrombus, infection in related organs, penetrating trauma or bacterial endocarditis, causing an aggressive local infection with rapid expansion of the arterial wall and a high risk of rupture ${ }^{1}$.

Mycotic thoracic aortic aneurysms (MTAA) account for less than $1 \%$ of all aneurysm repairs in the USA compared to up to $3 \%$ of all aneurysm repairs in Asia ${ }^{1,2,8}$. Symptoms are often nonspecific such as fever, sepsis and less frequently chest- or shoulder pain ${ }^{3}$. Tracheal compression due to a TAA is very rare ${ }^{9}$. Rupture or impending rupture occurs in $50-85 \%^{2,9}$. Diagnosis remains difficult with nonspecific laboratory findings such as leucocytosis in $65-85 \%$ or elevated C-reactive protein in $75-80 \% \%^{9}$. Blood cultures are positive in $50-90 \%$ but become negative in up to $25-50 \%$ after starting antibiotic treatment ${ }^{9}$. In the Western world the most common pathogens are streptococcal and staphylococcal species, gram negative bacteria such as Salmonella or tuberculosis have a high prevalence in $\mathrm{Asia}^{2,8}$. On CTA scan a saccular aneurysm with an irregular contour in absence of extensive atherosclerosis is suggestive of a mycotic aneurysm ${ }^{9}$. Periaortic findings such as soft tissue inflammation may also be present ${ }^{9}$.

Historically, surgical debridement, intravenous antibiotic treatment and open aorta repair is considered the treatment of choice. However, recent studies, i.e. an European multicentre study on mycotic aortic aneurysms showed durable outcome of endovascular treatment with $91 \%$ survival at 30 days, $19 \%$ fatal infection-related complications and 55\% 5-year survival rate ${ }^{5}$. Nevertheless, in patients with a ruptured aneurysm, fever, tracheal- or oesophageal fistula with or without uncontrolled bleeding at time of surgery, endovascular repair should be considered a bridge to definite surgical repair ${ }^{10}$.

In this case report, emergency TEVAR was regarded as designated treatment over open repair given the expertise in thoracic stent grafting at our institute and patient characteristics, in line with recent literature ${ }^{5,10}$. The clinical deterioration after primary intervention was considered the result of ongoing infection and progressive dilatation of the aneurysm, resulting in a new or, at completion angiography undiagnosed, type Ib endoleak. After primary airway management a second vascular intervention was considered. While, open aneurysm repair lacked patient compliance, endovascular extension of the stent was performed. Even though, the patient initially recovered after the endovascular stent extension and tracheal stent placement a new episode of respiratory insufficiency developed. Removing the tracheal stents to restore the trachea and thereby restoring the cough reflex and cilia function was considered. However, because of the prolonged and long anticipated hospitalization the patient wished no further invasive treatment. This severe course following unsuccessful endovascular treatment of a MTAA has not been described before and was lethal in the presented case.

\section{Conclusion}

This case report shows a rare complication following failed endovascular treatment, where growth of the saccular aneurysm in the presence of ongoing infection and type Ib endoleak resulted in severe compression of the trachea. Even though treatment of mycotic aneurysms improved due to antibiotic treatment and endovascular stent grafting, complications and graft failure do occur and can be lethal as presented in our case.

\section{References:}

1. Lau C, Gaudino M, de Biasi AR, Munjal M, Girardi LN. Outcomes of Open Repair of Mycotic Descending Thoracic and Thoracoabdominal Aortic Aneurysms. Ann Thorac Surg. 2015;100(5):1712-1717.

2. Oderich, GS, Panneton JM, Bower TC, Cherry KJ Jr, Rowland CM, Noel AA et al. Infected aortic aneurysms: aggressive presentation, complicated early outcome but durable results. J Vasc Surg . 2001; 34:900-908. 
3. Malouf JF, Chandrasekaran K, Orszula TA. Mycotic aneurysms of the thoracic aorta: a diagnostic challenge. The American Journal of Medicine. 2003;115(6):489-496.

4. Alsawas M, Zaiem F, Larrea-Mantilla L, Almasri J, Erwin PJ, Upchurch GR Jr, Murad MH. Effectiveness of surgical interventions for thoracic aortic aneurysms: A systematic review and meta-analysis. Journal of Vascular Surgery. 2017;66(4): 1258-68.

5. Wilson WR, Bower TC, Creager MA, Amin-Hanjani S, O'Gara PT, Lockhart PB et al. Vascular Graft Infections, Mycotic Aneurysms, and Endovascular Infections: A Scientific Statement From the American Heart Association. Circulation. 2016;134(20): e412-460.

6. Hiratzka LF, Bakris GL, Beckman JA, Bersin RM, Carr VF, Casey DE Jr. et al. ACCF/AHA/AATS/ACR/ASA/SCA/SCAI/SIR/STS/SVM guidelines for the diagnosis and management of patients with Thoracic Aortic Disease: a report of the American College of Cardiology Foundation/American Heart Association Task Force on Practice Guidelines, American Association for Thoracic Surgery, American College of Radiology, American Stroke Association, Society of Cardiovascular Anesthesiologists, Society for Cardiovascular Angiography and Interventions, Society of Interventional Radiology, Society of Thoracic Surgeons, and Society for Vascular Medicine. Circulation. 2010;121(13): e266-369.

7. Mathur A, Mohan V, Ameta D, Gaurav B, Haranahalli P. Aortic aneurysm. J Transl Int Med. $2016 ; 4(1): 35-41$.

8. Hsu RB, Tsay YG, Wang SS, Chu SH. Surgical treatment for primary infected aneurysm of the descending thoracic aorta, abdominal aorta, and iliac arteries. J Vasc Surg. 2002; 36:746-750.

9. Sörelius K, Mani K, Björck M, Sedivy P, Wahlgren CM, Taylor P et al. Endovascular treatment of mycotic aortic aneurysms: a European multicenter study. Circulation. 2014;130(24):2136-2142.

10. Kan CD, Lee HL, Yang YJ. Outcome after endovascular stent graft treatment for mycotic aortic aneurysm: a systematic review. J Vasc Surg. 2007;46(5):906-912.

\section{Figures}

Figure $1 \mathrm{~A}+\mathrm{B}$ thoracic aortic aneurysm with pending rupture

Figure 2 on angiography an endoleak type $1 \mathrm{~b}$ was suspected

Figure 3 completion angiography after proximal and distal stent extension, no signs of an endoleak

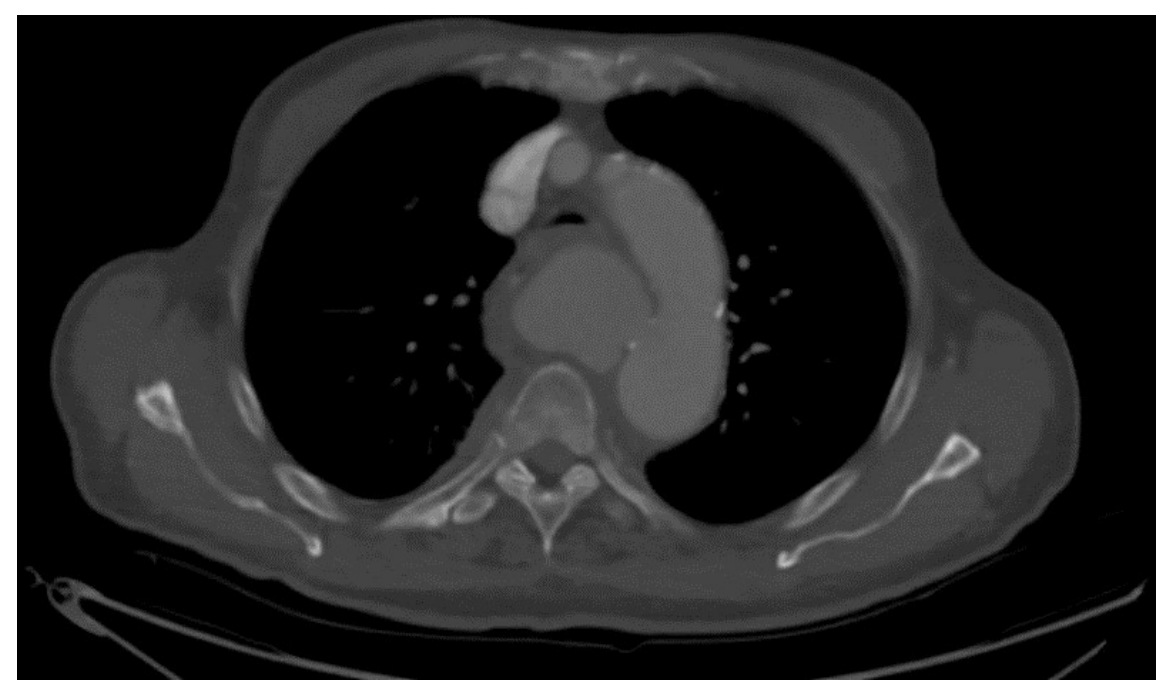



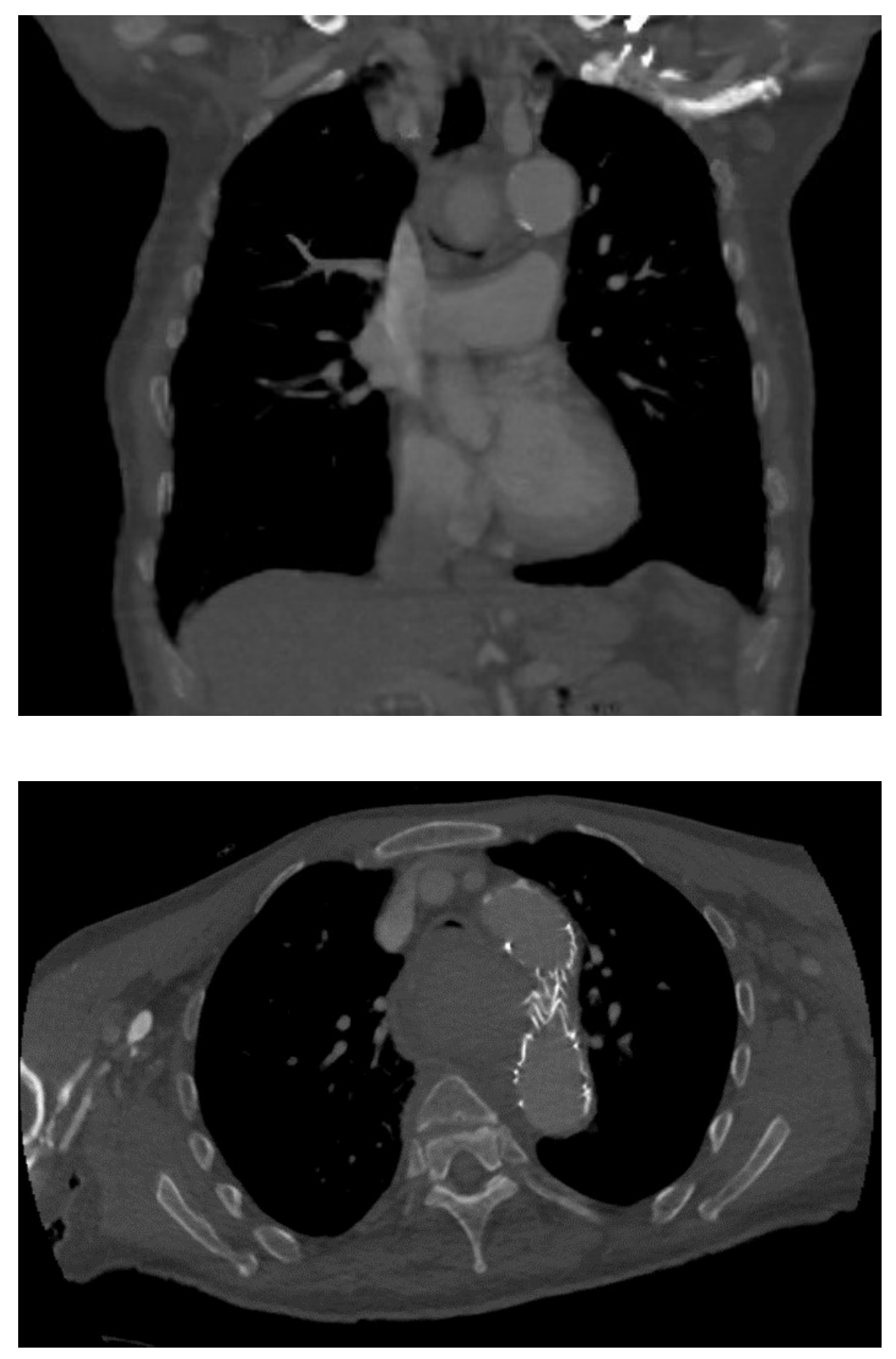


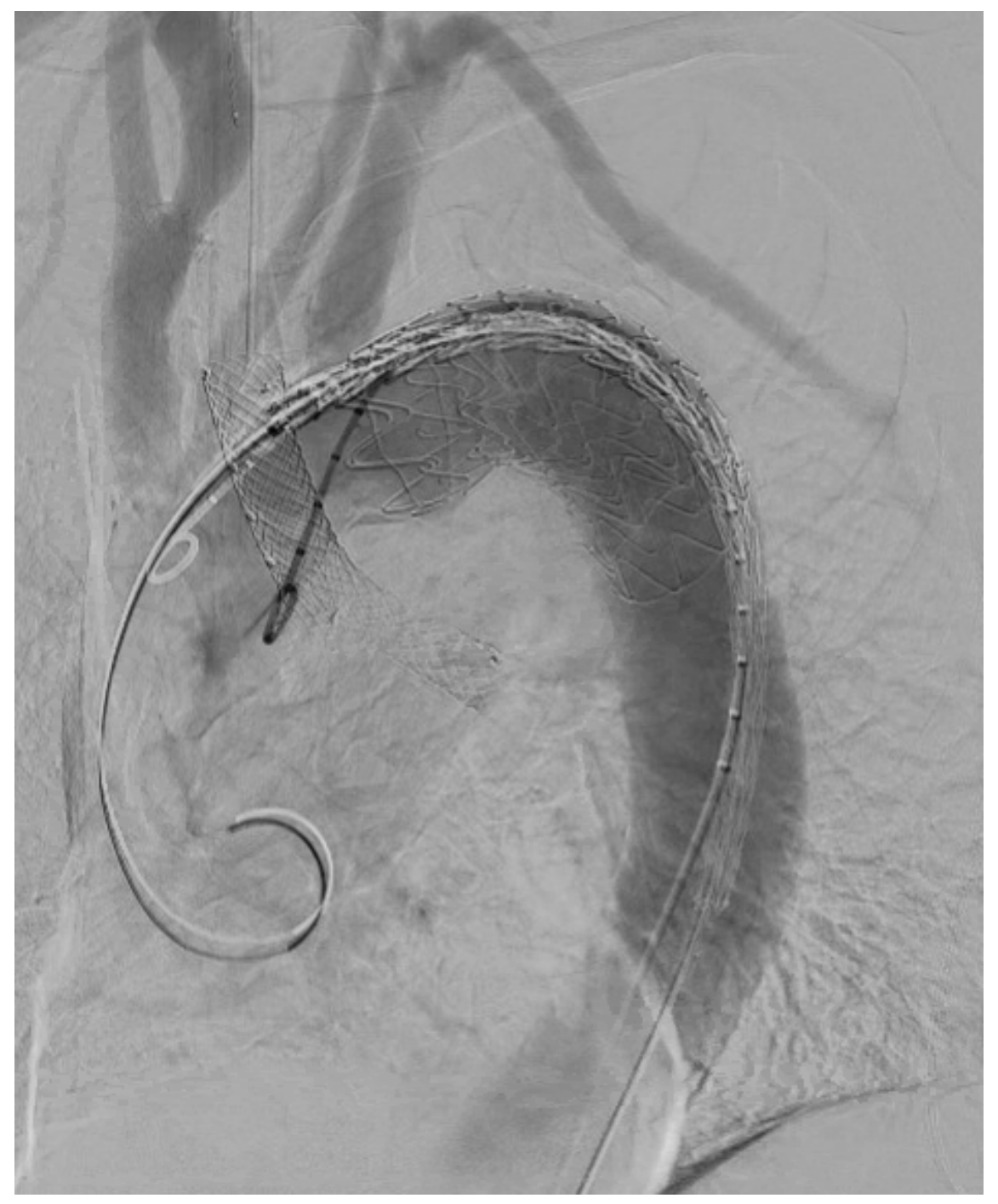




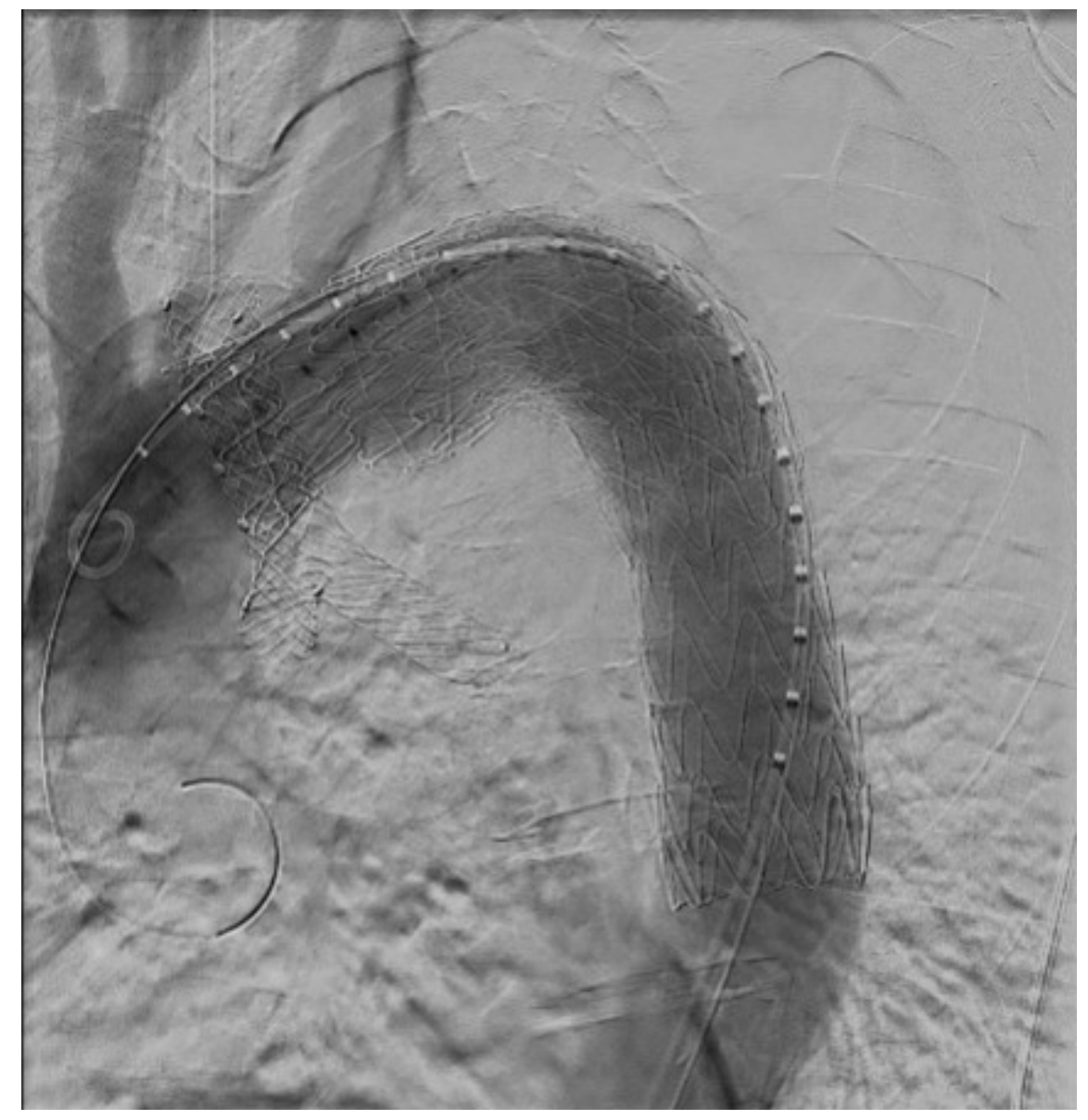

